PRONTOSIL ALBUM IN PUERPERAL SEPSIS BY

\author{
M. A. FOULIS, M.D. \\ Assistant Superintendent and Resident Surgeon
}

AND

JOHN B. BARR, M.B., Ch.B.

Senior Resident Medical Officer, Robroyston Hospital, Glasgow

A rapidly growing literature, from both the experimental and clinical aspects, proves beyond reasonable doubt that prontosil constitutes an invaluable measure in the treatment of severe streptococcal infections. Recent articles, both in this country and abroad, suggest that its usefulness may not be limited only to infections by haemolytic streptococci. In puerperal sepsis, however, the treatment of this streptococcal infection is of paramount importance, since it is responsible for almost all the deaths.

The following brief notes represent our experience of "prontosil album" (" the sulphonamide") in the treatment of puerperal infections. The use of this simpler "reduction" compound of prontosil seems logical in the light of recent experiments (Colebrook, et al., 1936), which demonstrate that the original compounds, prontosil soluble and prontcsil "red," are active against haemolytic streptococci only in so far as they are broken down in the body to the sulphonamide or some closely related substance. Certainly it has been shown that in vitro prontosil is not lethal to streptococci, whereas the sulphonamide, prontosil album, can destroy them under conditions of dilution fairly closely approaching those which might be encountered in the body.

\section{The Patients Treated}

Prontosil album was used in treatment, in a ward given over to puerperal sepsis, from November 1, 1936, and these notes may be regarded as covering the period from that date till January 31, 1937. During the period under review seventy patients, all proven septic, came into hospital for treatment. There was one death, giving a mortality rate of 1.4 per cent. The average death rate over 1,988 patients, all of whom were septic; treated between the years 1931 and 1936 was 13.5 per cent. The use of prontosil album was limited to twenty-two casesnamely, women who were severely ill, having repeated rigors, frank septicaemia, or peritonitis. This group comprised eleven with septicaemia, four with peritonitis, and seven others. Three patients each received up to $20 \mathrm{c.cm}$. prontosil soluble on occasion, owing to a temporary shortage of prontosil album. One other received $40 \mathrm{c.cm}$. prontosil soluble on each of three successive days.

The great drop which has occurred in the death rate raises prominently the possibility that the seventy cases under discussion were less severely ill than those treated in the past. It is agreed that the death rate in puerperal sepsis is closely bound up with the occurrence of septicaemia and peritonitis. In the present group there were eleven cases of septicaemia, eight of which yielded a growth of haemolytic streptococci on primary blood culture. The incidence of septicaemia in the whole group is therefore 15.7 per cent., the average incidence from 1931 till 1936 being 18.6 per cent. There were four instances of generalizing peritonitis without blood invasion, and on laparotomy three yielded cultures of haemolytic streptococci. The incidence of peritonitis of
5.7 per cent. is to be compared with 4 per cent., which was the average incidence over the five years ending in 1936.

It would seem, therefore, that while there has been a slight drop in the incidence of severe sepsis the fall in death rate must be explained by improved treatment or lessened virulence of the infecting organism.

\section{Dosage and Toxicity}

In the beginning the dosage used was small, but with increasing experience this was raised to 7.2 grammes (twentyfour tablets of 0.3 gramme) spread over twenty-four hours for averagely ill patients. This dosage, it was discovered, could be continued for seven to ten days without harm. It was found advisable to continue medication for two to three days after subsidence of the fever. Increase in the dosage in more severely ill patients was almost always accompanied by a corresponding clinical improvement. In one very severe case of septicaemia a daily consumption of 14.4 grammes (forty-eight tablets) for four successive days and 7.2 grammes for a further three days was followed by nothing worse than a slight cyanosis, possibly attributable to sulphaemoglobinaemia. Another case of septicaemia received $40 \mathrm{c.cm}$. prontosil soluble and 10.8 grammes prontosil album for three successive days and 7.2 grammes each day for a further two days, with again nothing worse than slight cyanosis, possibly accounted for by the pneumonic septicaemia, which demanded such heroic dosage. Both cases recovered. In one instance it was noticed that a daily dosage of 10.8 grammes was ineffective till 15 minims of dilute hydrochloric acid were given, somewhat empirically, before ingestion of the tablets.

From the remarks above it is obvious that the toxicity of prontosil album is low. Sickness and vomiting occurred in one case after a total dosage of 75 grammes spread over nine days, and ceased on discontinuing the drug. The urine from several highly dosed patients showed no casts, but mild albuminuria appeared twice where it had not hitherto been present. In three patients difficulty in micturition developed, amounting almost to retention of urine. There was no oliguria. In no case was jaundice recorded, nor were there nervous symptoms. Sulphaemoglobinaemia was noticed as a possibility in the two cases already mentioned when dosage was discussed. In this respect it may be mentioned that magnesium sulphate, which, it is thought, may have some causal influence, was given in one case only. In the other the probability of sulphur compounds being present in commercial glucose cannot be ignored.

A much larger number of patients have been treated with the sulphonamide, prontosil album, than with the original injection preparation, prontosil soluble. So far as the death rate gives any indication the two drugs are apparently of equal value. The obvious advantages of an easily ingested preparation over large and sometimes painful injections need only be mentioned. Some of the temperature charts reviewed suggest that the action of prontosil album is more rapid than that of prontosil soluble, even allowing for the quicker absorption of the latter. On present knowledge of the pharmacology of these drugs it would appear reasonable to combine the two preparations, the injections acting perhaps as a depot of continuous sulphonamide therapy. In severe peritonitis the ingestion of large numbers of tablets, possibly roughly chewed by the patient, and the swallowing of considerable amounts of fluid may be open to objection. 


\section{Case Reports}

Below are set out the abbreviated records of three cases illustrative of the type of result obtained. The first case is one of septicaemia with peritonitis, the second one of what appears to have been, despite the absence of confirmatory blood culture, septicaemia associated with multiple bacterial emboli. The third case is one of moderate severity, and is quoted to complete the clinical picture.

Case 1.-A 5-parous woman aged 30 years was confined on October 30,1936, when the perineum was badly torn. She sickened on November 1 with rigors, cough, and abdominal pain, and was admitted on November 3. On admission the temperature was $104^{\circ} \mathrm{F}$., the pulse rate 128 , and the respirations 34 a minute. She was very pale, and had a gross patchy bilateral bronchopneumonia with extensive pleural friction. Peritonitis was present up to a level higher than the umbilicus. A blood culture which was taken on admission yielded a liberal growth of haemolytic streptococci. An immediate laparotomy revealed a generalizing peritonitis, which was drained. The sero-pus yielded numerous colonies of haemolytic streptococci. She was given 0.9 gramme prontosil album four-hourly (three tablets) as soon as she could swallow. The following day her peak temperature was $101^{\circ}$ and within forty-eight hours was normal. Prontosil album was continued for five days at the same dosage. The convalescence was interrupted by the development of phlegmasia alba dolens.

Case 2.-A woman aged 31 years, 2-para, was confined on December 30,1936. There was no gross trauma, but she sickened on January 5, 1937, with rigors, headache, and a scarlatiniform rash. We admitted her on January 11 with patchy bronchopneumonia and a widespread scarlatiniform rash tending to vesiculation on the face and soft palate. There were numerous acutely inflamed painful indurated areas beneath the skin on the arms, knuckles, and legs. There was possibly early effusion into the right elbow- and the left anklejoint. Blood culture was negative, but the patient was undoubtedly septicaemic. At the beginning of therapy by prontosil album 1.8 grammes (six tablets) four-hourly her temperature was $103.2^{\circ}$, pulse rate 128 , and respirations 24 a minute. She was slightly cyanosed. Allowing for doses missed during sleep she received a total of 250 tablets $(75$ grammes) prontosil album in four-hourly doses over nine days, by which time the illness was over. The rash, induration, and vesicles retrogressed on the fourth day of treatment, and the joints, once thought to be the seat of septic arthritis, were free of pain and swelling on the seventh day. One larger induration, which became semi-fluctuant and from which it was hoped to culture an organism, was totally absorbed.

Case 3.-A girl aged 19 years was confined on December 8, 1936. A persistent occipito-posterior presentation resulted in a severe perineal tear. She sickened on December 10 with an erythematous rash and a rise in temperature to $107^{\circ}$ and a pulse rate of 160 a minute. She was immediately given two tablets of prontosil album, and this was repeated in four hours. She was admitted to Robroyston Hospital on December 10 with a temperature of $99^{\circ}$, pulse rate 104 , and respirations 24 a minute. Her symptoms were suggestive of acute metritis. She was given prontosil album, two tablets $(0.6$ gramme) four-hourly for four days, after which the temperature was normal. A blood culture, taken on admission, yielded scanty haemolytic streptococci.

\section{Summary}

1. Out of a series of seventy cases of puerperal sepsis twenty-two were treated with the "sulphonamide" preparation prontosil album. Four of these had, in addition, occasional doses of prontosil soluble by injection. The other forty-eight cases were not considered sufficiently serious for chemotherapy.

2. Eleven of the twenty-two cases treated with prontosil album had septicaemia, eight proved by blood culture to be due to haemolytic streptococci, and a further three cases of peritonitis without septicaemia all yielded haemolytic streptococcal colonies on culture of the peritoneal fluid. The balance-seven cases-showed a haemolytic streptococcus on bacteriological examination of the vaginal discharge.

3. The dosage of prontosil album was high, ranging from 3 grammes (ten tablets) to 14.4 grammes every twenty-four hours, but the preparation was well tolerated and toxic effects were minimal.

4. The fall in temperature and the general improvement were rapid and striking.

5. The success of the treatment is evidenced in the mortality rate of 1.4 per cent. (one death) in the whole series, as compared with 13.4 per cent. in a five-year period in the same hospital.

Colebrook, et al. (1936). Lancet, 2, 1323.

\section{Clinical Memoranda}

\section{Method of Removing Wax Foreign Bodies from the Bladder}

From time to time reports are published of the removal of pieces of wax from the urinary bladder by means of rongeur forceps, lithotrites, or even by suprapubic cystotomy. (One transatlantic clinician recently reported how a patient had succeeded in voiding a piece of wax by urinating while suspended upside down from a trapeze.) It does not appear to be sufficiently widely recognized that a number of wax solvents, such as xylol, will deal with this type of case with a minimum of discomfort to the patient. Hattinger was the first, I believe, to describe the use of xylol for this purpose.

I have recently come across two cases in which wax had been introduced by a male patient along the urethra for erotic purposes, and, as so often happens, the distal portion of the improvised wax bougie broke off, the remainder entering the bladder, whence it did not escape, and set up an inflammatory reaction.

The first case, a boy of 16 , was brought to me a few days after the "incident" had occurred. He was suffering from frequent and painful micturition, and no attempt was made to hide the origin of the trouble. Cystoscopy revealed the presence in the dome of the bladder of a flattened piece of wax about the diameter of a shilling. The cystoscope was withdrawn, a catheter passed, and the bladder filled with 6 ounces of sterile water; $10 \mathrm{c} . \mathrm{cm}$. of xylol was then introduced, and the catheter spigoted. The catheter was left in position for two hours, the suprapubic region being kneaded at frequent intervals to aid the solution of the wax in the xylol. On removal of the spigot the bladder emptied, the terminal few cubic centimetres being a white milky substance, representing the wax in solution. A cystoscope was again introduced, and no longer was there any evidence of the wax.

A few weeks later a similar case came into All Saints' Hospital under the care of my colleague, Mr. F. McG. Loughnane. The history in this case did not give any inkling as to the cause of the dysuria, but cystoscopy revealed the presence of a piece of wax in the dome of the bladder. I related my experience in the first case and the same procedure was adopted in this second one with complete success. 